\title{
Extracellular Vesicles in HIV, Drug Abuse, and Drug Delivery
}

\author{
Santosh Kumar ${ }^{1} \cdot$ Nazira El-Hage ${ }^{2}$ • Elena Batrakova ${ }^{3}$
}

Received: 10 July 2020 / Accepted: 15 July 2020 / Published online: 22 July 2020

\begin{abstract}
Extracellular vesicles (EVs) are known to perform important biological functions and have been implicated in multiple disease pathogeneses, including HIV and drugs of abuse. EVs can carry biological molecules via biofluids such as plasma and cerebrospinal fluids (CSF) from healthy or disease organs to distant organs and deliver biomolecules to recipient cells that subsequently alter the physiology of the recipient organs. As biocarriers, EVs have the potential to be developed as non-invasive biomarkers for disease pathogenesis and drug abuse, as the level of specific EV components can be altered under disease/drug abuse conditions. Since many drugs don't cross the blood-brain barrier, EVs have shown the potential to encapsulate small drug molecules, including nucleotides, and carry these drugs to brain cells and enhance brain drug bioavailability. Through this special issue, we have covered several studies related to the role of EVs in altering biological functions via cell-cell interactions in healthy, HIV, and drug of abuse conditions. We have also included studies on the role of EVs as potential biomarkers for HIV pathogenesis and drugs of abuse. Further, the potential role of EVs in drug delivery in the CNS for diseases, including HIV-associated neurocognitive disorders and other neurological disorders, are covered in this issue.
\end{abstract}

Keywords Extracellular Vesicles $\cdot$ HIV $\cdot$ Drug abuse $\cdot$ Drug delivery $\cdot$ HAND $\cdot$ Biomarkers

Extracellular vesicles (EVs), especially exosomes, are nanovesicles that have been recognized as valuable targets for biological research. Although EVs were first isolated and characterized in the late 1980 s, their potential in biological research was not realized until a decade ago. EVs are produced by most cell types and are secreted into biofluids such as plasma, cerebrospinal fluids (CSF), and saliva, and transport their molecular cargos between diverse cell types at distant organs via these fluids. These qualities make them key mediators in intercellular communication both in healthy, disease, and other conditions, e.g. drug abuse. Based on the triggers and physiological conditions of the cells/organs, EVs

Santosh Kumar

ksantosh@uthsc.edu

1 Department of Pharmaceutical Sciences, College of Pharmacy, University of Tennessee Health Science Center, 881 Madison Ave, Memphis, TN 38163, USA

2 Department of Immunology and Nanomedicine, Herbert Wertheim College of Medicine, Florida International University, Miami, FL, USA

3 Center for Nanotechnology in Drug Delivery, Eshelman School of Pharmacy, University of North Carolina at Chapel Hill, Chapel Hill, NC, USA may carry toxic or protective components, which upon delivering to the recipient cells/organs, may cause cytotoxicity/ organ damage or cellular/organ protection, respectively.

The semi-selective ability to package and transport diverse biological cargos such as proteins, miRNAs, lipids, and small molecules, allow EVs to serve as diagnostic biomarkers of disease states and other conditions. Due to their relative stability, availability in biofluids, and stimulus-inducible cargos, EVs are ideal candidates for biomarkers of many diseases, including HIV and drugs of abuse conditions. In addition, due to their natural ability to transport biomolecules, EVs have been studied for potential drug delivery systems for therapeutic interventions. This special issue features several papers that highlight the role of EVs in drug delivery modalities, especially across the blood-brain barrier (BBB), for the interventions of many CNS diseases, including HIV and HIVAssociated Neurocognitive Disorder (HAND).

Although, over the years, multiple EV isolation and purification methods have been developed, it is very difficult to separate EVs from viruses due to the similar sizes. Thus, it is imperative to separate these two populations to avoid misinterpretations of data. Here, McNamara and Dittmer described modern methods that combine ultracentrifugation, cross flow filtration, column chromatography, including affinity chromatography, as well as NanoFACS and Flow 
Virometry (McNamara and Dittmer 2019). Depending upon the contaminant viruses, such as human immunodeficiency virus (HIV), hepatitis A, or influenza, as described in the paper, a combination of these methods can be used. International Society of Extracellular Vesicles (ISEV) 2018 guidelines recommend optimal use of isolation method(s) based on the source and application of EVs. Thus, different methods or a combination of methods can be used in different studies depending upon the source or outcome measures of the EVs in that study.

EVs transported from peripheral or stem cells via plasma can infiltrate into the CNS and deliver their components to the brain cells causing physiological changes. In a study presented here, Branscome et al. have shown that induced pluripotent stem cell(iPSC) and mesenchymal stem cell- (MSC) derived EVs display immunomodulatory properties with distinct mechanisms in CNS cells (Branscome et al. 2019). They have shown the potential of these EVs in repairing damaged cells. The study has implications with regard to the role of iPSC-and MSC-derived EVs in therapeutic interventions for CNS diseases in which brain cells are damaged as a result of stimuli.

Both HIV and drug abuse stimulate specific cell types/ organs to secrete EVs containing specific components. As reviewed by Rahman et al., alcohol exposure modifies the contents of hepatic and extra-hepatic EVs, which translocate via plasma, and deliver their cargos to non-hepatic cells and alter inflammatory responses (Rahman et al. 2019). Some of the components that are elevated upon alcohol exposures are the alcohol-metabolizing cytochrome P4502E1 enzyme, antioxidant enzymes, oxidative stress-inducing miRNAs, and cytokines and chemokines. However, the underlying mechanism of packaging of these components and their specific roles in recipient cells are poorly known. Thus, a complete understanding of the interplay between EVs and alcohol is necessary to develop EVs as biomarkers for alcohol or other druginduced pathogeneses. In a study by Wang et al., the dysregulation of plasma exosomal miRNAs by HIV infection and/or heroin use, was demonstrated (Wang et al. 2019). However, the source of EVs containing these miRNAs is still under investigation. Thus, in addition to investigating the source of these plasma EVs, it is imperative to study whether dysregulated EV miRNAs play a role in HIV and/or heroin usemediated immune dysfunction and neurotoxicity. In a separate study, Hu et al. demonstrated the role of the HIV-1 protein Tat in induction and release of EV miRNA-7 from astrocytes ( $\mathrm{Hu}$ et al. 2019). The EV miRNA-7 is taken up by neurons and downregulates neuronal neuroligin 2 (NLGN2), ultimately altering synaptic functions. This is another important finding with respect to the role of HIV in inducing EV cargos that alter disease physiology by cell-cell interactions.

Autophagy-exosome interplay, which is generally triggered by oxidative stress, causes both survival and cell death based on the intensity/dose or exposure time of a stimuli. Here,
Olanrewaju and Hakami reviewed the implications of autophagy-exosome interplay for microglial response to HIV infection within the CNS (Olanrewaju and Hakami 2020). They report the involvement of specific protein complexes that contain autophagy-related (ATG) proteins, such as ATG101, ATG13, FIP200, in exosome packaging during infection, or the influence of exosome-associated tetraspanins, e.g. CD63 and CD81, on autophagy response during infection. ATG101, ATG13 and FIP200 are also involved in the phagophore formation and elongation processes, which are essential in the autophagy pathway. The current investigation of exosome-autophagy crosstalk in microglia in the PD system is important, as microglial function is also implicated in HIV and neuroAIDS.

EVs and their specific components, obtained from biofluids such as plasma, urine, and saliva, are considered important non-invasive diagnostic markers for cancers and other diseases/conditions. In one of the studies presented here, Kodidela et al. identified specific plasma EV proteins that were altered in HIV + individuals, however, different proteins were altered in HIV-infected drinkers or smokers (Kodidela et al. 2019). For example, hemopexin was not altered in drinkers or HIV + individuals, but was altered in HIV + drinkers. Additionally, properdin was not altered in HIV + or smoker groups, but was altered in HIV + smokers. These results suggest an important role for hemopexin and properdin in HIV-alcohol and HIV-tobacco interactions, respectively. Since drug abuse is known to exacerbate HIV pathogenesis and HAND, it is of great significance to develop diagnostic markers for HAND in HIV populations who also abuse drugs such as alcohol, tobacco, cocaine, and opioids.

Due to the small size and lipophilic nature of EVs, they are increasingly being exploited as delivery vehicles of therapeutics for anticancer strategies, immunomodulation, targeted drug delivery, tissue regeneration, and vaccination. In the review by Sil et al., they highlight the pros and cons of using EVs as conduits of proteins, lipids, carbohydrates, mRNAs, long non-coding RNAs, microRNAs and DNAs for therapeutic agents (Sil et al. 2019). Efficient targeted delivery of anticancer agents to cancer cells remains one of the greatest challenges to developing therapies. In the article by Haney et al., they exploit the natural ability of macrophages to target cancer cells by means of EVs as drug delivery vehicles for the chemotherapeutic agents, paclitaxel (PTX) and doxorubicin (Dox) (Haney et al. 2019). Using a mouse model of pulmonary metastases, they demonstrated that macrophage-derived EVs loaded with PTX (EV-PTX) and Dox (EV-Dox) target cancer cells and exhibit high anticancer efficacy. As a nice follow-up, in the review article by Shahjin et al., they discussed the challenges faced in EV research as it relates to HIV-1 co-morbid conditions and the potential of nanotechnologies, especially EVs, in Neuro-AIDS (Shahjin et al. 2019). The structure of the BBB, the presence of efflux pumps, and the expression of metabolic enzymes, pose hurdles for drug- 
brain entry. With only a limited number of drugs, including small lipophilic compounds, transmigrating the BBB, drug delivery to the CNS has become a challenge and much effort has been invested into improving the route of drugs to the brain. In the article by Reynolds and Mahajan, they tackled another important issue related to the development of targetspecific therapy in the brain (Reynolds and Mahajan 2019). They used an artificial BBB model system to investigate the transmigration of microglia-derived exosomes containing siRNA targeting Tetraspanin (Tspan)-2, expressed on microglia cells. Successful transmigration of the exosomes across the BBB was evaluated by the knockdown of Tspan 2 and by the modulation of neuroimmune responses in HIV-infected microglial cells plated on the basal side of the BBB. In the article by Liao et al., they bypassed the BBB and intranasally delivered EVs loaded with Long non-coding RNAs (lncRNAs) in the brains of mice (Liao et al. 2019). Intranasal delivery is a non-invasive method to deliver drugs to the brain that has been proven safe and effective.

As described in this issue, the understanding of the role of EVs as natural carriers of biomolecules and the use of EVs as drug delivery vehicles offers a number of benefits over common drug administration regimens. However, limitations and challenges still exist that need to be addressed in future research to improve the development of drug delivery strategies aided in saving lives.

Acknowledgements We acknowledge all the contributors of this special issue and we are grateful to the reviewers who provided comments and suggestions to the manuscripts and helped strengthen the content of the special issue.

\section{Compliance with Ethical Standards}

Conflict of Interest The authors declare no competing interests.

\section{References}

Branscome H, Paul S, Khatkar P et al (2019) Stem cell extracellular vesicles and their potential to contribute to the repair of damaged
CNS cells. J Neuroimmune Pharmacol. https://doi.org/10.1007/ s11481-019-09865-y

Haney MJ, Zhao Y, Jin YS et al (2019) Macrophage-derived extracellular vesicles as drug delivery systems for Triple Negative Breast Cancer (TNBC) therapy. J Neuroimmune Pharmacol. https://doi.org/10. 1007/s11481-019-09884-9

Hu G, Niu F, Liao K et al (2019) HIV-1 tat-induced astrocytic extracellular vesicle miR-7 impairs synaptic architecture. J Neuroimmune Pharmacol. https://doi.org/10.1007/s11481-019-09869-8

Kodidela S, Wang Y, Patters BJ et al (2019) Proteomic profiling of exosomes derived from plasma of HIV-infected alcohol drinkers and cigarette smokers. J Neuroimmune Pharmacol. https://doi.org/ 10.1007/s11481-019-09853-2

Liao K, Niu F, Dagur RS et al (2019) Intranasal delivery of lincRNACox 2 siRNA loaded extracellular vesicles decreases lipopolysaccharide-induced microglial proliferation in mice. $\mathrm{J}$ Neuroimmune Pharmacol. https://doi.org/10.1007/s11481-01909864-z

McNamara RP, Dittmer DP (2019) Modern techniques for the isolation of extracellular vesicles and viruses. J Neuroimmune Pharmacol. https://doi.org/10.1007/s11481-019-09874-x

Olanrewaju AA, Hakami RM (2020) The messenger apps of the cell: extracellular vesicles as regulatory messengers of microglial function in the CNS. J Neuroimmune Pharmacol. https://doi.org/10. 1007/s11481-020-09916-9

Rahman MA, Patters BJ, Kodidela S, Kumar S (2019) Extracellular vesicles: intercellular mediators in alcohol-induced pathologies. $\mathrm{J}$ Neuroimmune Pharmacol. https://doi.org/10.1007/s11481-01909848-z

Reynolds JL, Mahajan SD (2019) Transmigration of tetraspanin 2 (Tspan2) siRNA via microglia derived exosomes across the blood brain barrier modifies the production of immune mediators by microglia cells. J Neuroimmune Pharmacol. https://doi.org/10.1007/ s11481-019-09895-6

Shahjin F, Chand S, Yelamanchili SV (2019) Extracellular vesicles as drug delivery vehicles to the central nervous system. J Neuroimmune Pharmacol. https://doi.org/10.1007/s11481-01909875-w

Sil S, Dagur RS, Liao K et al (2019) Strategies for the use of extracellular vesicles for the delivery of therapeutics. J Neuroimmune Pharmacol. https://doi.org/10.1007/s11481-019-09873-y

Wang X, Sun L, Zhou Y et al (2019) Heroin abuse and/or HIV infection dysregulate plasma exosomal miRNAs. J Neuroimmune Pharmacol. https://doi.org/10.1007/s11481-019-09892-9

Publisher's Note Springer Nature remains neutral with regard to jurisdictional claims in published maps and institutional affiliations. 